\title{
Pneumologie: modern, mit langer Tradition
}

\author{
Pneumology - Modern, with Tradition
}

Autor

Institut
Michael Pfeifer

Klinik Donaustauf, Universität Regenburg
Bibliografie

DOI http://dx.doi.org/

10.1055/s-0029-1243944

Pneumologie 2010; 64:

141-142 @ Georg Thieme

Verlag KG Stuttgart · New York

ISSN 0934-8387

Korrespondenzadresse

Prof. Dr. med. Michael Pfeifer

Klinik Donaustauf

Universität Regenburg

Ludwigstr. 68

93093 Donaustauf

Michael.Pfeifer@klinik.

uni-regensburg.de
Weltweit sind die Erkrankungen der Lunge eine der führenden Ursachen für Morbididtät und Mortalität. Global betrachtet, sind 19\% aller Todesfälle und 15\% der „disabilty-adjusted life years“ (DELYs) [1] direkt eine Folge von Erkrankungen der Atmungsorgane. Auch wenn Lungenkrankheiten schon immer eine große Rolle eingenommen haben, ist ihre Bedeutung in den letzten Jahrzehnten erheblich gewachsen, was sich in einer stetig zunehmenden Inzidenz und Prävalenz zeigt. Dies ist in erster Linie eine Folge der erheblichen Zunahme der wichtigsten Risikofaktoren einschließlich der allgemeinen Urbanisierung und Industrialisierung, sprich des Tabakgenusses, der Umweltbelastung und der HIV-Epidemie. Eine wesentliche Bedeutung für die Zunahme der Lungenerkrankungen hat aber auch die demografische Entwicklung der letzten Jahrzehnte mit einer wachsenden Lebenserwartung breiter Bevölkerungsschichten. Diese globale Entwicklung spiegelt sich letztlich mit unterschiedlichen Schwerpunkten auch in Deutschland wider.

Wie in den meisten europäischen Nachbarländern hat sich die deutsche Pneumologie aus der klassischen Phthisiologie entwickelt. Die Tuberkulose war über die praktische Medizin hinaus die große gesundheitspolitische Herausforderung in der Zeit der fortschreitenden Industrialisierung des ausgehenden 19. und frühen 20. Jahrhunderts. Entsprechend war die Bedeutung der Lungenmedizin in dieser Zeit sehr hoch, was zu einer flächendeckenden Versorgung des Landes mit Lungenkliniken abseits von den Städten, in landschaftlich schöner Umgebung führte. Diese im internationalen Vergleich erfolgreiche und vorzeigbare Infrastruktur hat jedoch bekanntermaßen die Entwicklung einer akademischen und universitären Pneumologie in Deutschland behindert, sodass das Fach Pneumologie zu den „kleinen“ Fächern innerhalb der Inneren Medizin gerechnet wird. Die Krankheitszahlen weltweit, aber auch hier in Deutschland sprechen jedoch eine andere
Sprache mit einer wachsenden Nachfrage nach pneumologischer Versorgung. Es ist bemerkenswert, dass diese Entwicklung noch nicht in den akademischen Zirkeln der medizinischen Fakultäten erkannt wurde, wie die aktuellen Vorkommnisse in Ulm zeigen, wo das Berufungsverfahren für einen pneumologischen Lehrstuhl kurz vor dem Abschluss ohne Begründung eingestellt wurde. Die Einführung des DRG-Systems hat die notwendige Transparenz geschaffen und gezeigt, welchen Stellenwert Lungenerkrankungen bei den stationären Behandlungsfällen haben [2]. Die sichtbare Konsequenz daraus ist die Einrichtung von zunehmend mehr eigenständigen pneumologischen Kliniken und Abteilungen in den Versorgungsstrukturen. So wurden allein in München und Umgebung in den letzten zwei Jahren fünf neue selbstständige pneumologische Abteilungen geschaffen.

Diese Entwicklung ist für die deutsche Pneumologie und insbesondere für die wissenschaftliche Fachgesellschaft eine Herausforderung und Chance zu gleich, wie der Artikel von Teschler et al. [3] in dieser Ausgabe der Pneumologie anlässlich des 100-jährigen Geburtstages der Deutschen Gesellschaft für Pneumologie und Beatmungsmedizin facettenreich darstellt. Ausgehend von einer Beschreibung und Analyse des Status quo zeigen die Autoren eine Vielzahl von Entwicklungsfeldern auf, die jetzt schon einen Stellenwert haben oder in den nächsten Jahren eine wichtige Bedeutung erlangen werden. In der Analyse des „Jetzt“ wird klar herausgearbeitet, dass die deutsche Pneumologie mehr Defizite aufweist als nur die immer wieder diskutierte fehlende Repräsentanz in den Universitäten. Es fehlt an Nachwuchs, an Ausbildungsstellen und im internationalen Vergleich an einer der Größe des Landes adäquaten Rolle in der klinischen Forschung. Aber es fehlt auch an einer entsprechenden Wahrnehmung der Pneumologie und der Probleme der Lungenerkrankungen in der Öffentlichkeit, was wieder- 
um von immenser Wichtigkeit für die politische Umsetzung der notwendigen Strukturmaßnahmen ist. Die Autoren weisen vor allem auf die große Herausforderung hin, eine breite und im Vergleich zu den anderen Fächern starke wissenschaftliche Basis in der deutschen Pneumologie aufzubauen. Zu Recht sehen die Autoren daher eine der wichtigsten Aufgaben der wissenschaftlichen Fachgesellschaft darin, diese Entwicklung neben der Sicherstellung des Fortbildungsauftrages zu unterstützen und gezielt Impulse zu geben. In Deutschland arbeitet die klinische Pneumologie auf einem sehr hohen Niveau und findet sich im Vergleich zu anderen Ländern in der Spitzengruppe wieder. Allerdings warten so viele Innovationen aus der Grundlagenforschung auf eine Translation in die Klinik, was nur bei Vorhandensein einer entsprechenden wissenschaftlichen Infrastruktur zu leisten ist. Es ist sicher richtig, dass Teschler et al. fordern, dafür die Pneumologie noch stärker in den Universitäten zu vertreten. Das allein wird jedoch nicht reichen, vielmehr muss auch in den großen Versorgungshäusern eine wissenschaftsorientierte Kultur weiterentwickelt werden, wie es ja schon in einigen klinischen Zentren gelungen ist.

Pneumologie ist in allen Bereichen der Medizin, von der Grundlagenforschung bis hin zu hoch technischen Anwendungen in der interventionellen Bronchologie oder Intensivmedizin, modern und spannend. Die Lungenheilkunde, die Pneumologie, ist auf einem sehr guten Weg, sich von einem Randgebiet wieder zu einem der großen Teilgebiete innerhalb der Inneren Medizin zu entwickeln, so wie sie einmal vor über 100 Jahre gestartet ist. Um auf diesem Weg erfolgreich zu sein, bedarf es jedoch noch vieler Anstrengungen und eines langen Atems.

Hinzu kommt noch eine unübersichtliche Zergliederung der Pneumologie in Vereinigungen, Arbeitsgemeinschaften, Netzwerke und Gesellschaften. Die Wahrnehmung der Pneumologie in der Öffentlichkeit gewinnt dadurch keine klaren Konturen, zu verwirrend und diffus ist ihre Darstellung in Lungentag, Atemwegsliga, Deutsche Lungenstiftung, DGP, BDP und viele andere Bereiche.

Das von den großen internationalen Gesellschaften ausgerufene „Year of the lung“ bietet jetzt die Chance, die deutsche Pneumologie organisatorisch so zu reformieren, dass in konzentrierten und gemeinsamen Aktionen die Bedeutung der Lungenerkrankung für die Gesundheit in der Öffentlichkeit erfolgreich und nachhaltig dargestellt werden kann.

\section{Literatur}

1 Murray CJ, Lopez AD. Alternative projections of mortality and disability by cause 1990-2020: Global Burden of Disease Study. Lancet 1997; 349: $1498-1504$

2 Beske, F, Katalinic A, Peters E, Pritzkuleit R. Morbiditätsprognose 2050. Ausgewählte Krankheiten für Deutschland, Brandenburg und Schleswig-Holstein. Kiel: IGSF, 2009

3 Teschler H, Seeger W, Vogelmeier C. Die Lage der Pneumologie in Deutschland: Status quo und Blick in die Zukunft. Pneumologie 2010; 64: $141-146$ 